\title{
One-dimensional deep learning architecture for fast fluorescence lifetime imaging
}

\author{
Dong Xiao, Yu Chen, and David Day-Uei Li
}

\begin{abstract}
We present a hardware-friendly deep learning architecture with one-dimensional convolutional neural networks (1D CNN) for fast analyzing fluorescence lifetime imaging (FLIM) data. A 1D CNN shows unparalleled advantages; they are more straightforward, quicker to train, and faster than high dimensional CNNs. 1D CNNs can be easily applied to multi-exponential fluorescence decay models. Compared with traditional least-square methods, superior performances of $1 \mathrm{D}$ CNNs on fluorescence lifetime image reconstruction have been validated using simulated data. We also employ the proposed 1D CNN to analyze two-photon FLIM images of functionalized gold nanoprobes in Hek293 and human prostate cancer cells. The results further demonstrate that $1 \mathrm{D}$ CNNs are fast and can accurately extract lifetime parameters from fluorescence signals. Our study shows that $1 \mathrm{D}$ CNNs have great potential in various real-time FLIM applications.
\end{abstract}

Index Terms-Fluorescence, Microscopy, Machine learning, Image analysis, Fluorescence lifetime imaging

\section{INTRODUCTION}

$\mathrm{F}$ LUORESCENCE lifetime imaging microscopy (FLIM) is a powerful tool for broad applications in molecular biology, analytical chemistry, and pharmacy; it can provide abundant information about cellular environments in living cells [1]. FLIM offers a unique quantitative way for monitoring intracellular parameters that are unattainable in intensity-based measurements, including ion concentrations, $\mathrm{pH}$, and temperature [2], [3]. It is also insusceptible to experimental conditions such as optical setups and fluorophore concentrations. FLIM has been widely applied in drug delivery and drug discovery. FLIM of autofluorescence can also be used for cancer diagnosis [4]. Also, FLIM offers a straightforward way to assess Förster Resonance Energy Transfer (FRET) behaviours. FLIM-FRET is a vital technique for investigating metabolic sensing, protein-protein interactions, and protein conformational changes [5] [6]. Measurements of fluorescence lifetimes can be time-domain or frequency-domain based. Among them, the time-correlated single-photon counting (TCSPC) method has been the gold standard due to its superior

Manuscript received May 18, 2020. This work was supported in part by the Medical Research Scotland (MRS-1179-2017) and Photon Force, Ltd., Edinburgh. (Corresponding author: David Li)

Dong Xiao and David Li are with the Strathclyde Institute of Pharmacy and Biomedical Sciences, University of Strathclyde, Glasgow, G4 0RE, Scotland, UK. (e-mail: dong.xiao@strath.ac.uk, david.li@strath.ac.uk )

Yu Chen is with the Department of Physics, University of Strathclyde, Glasgow, G4 0RE, Scotland, UK (e-mail: y.chen@ strath.ac.uk ). performances in the signal-to-noise ratio and temporal resolution [7]. In a TCSPC system, fluorophore-tagged samples are repetitively excited by an ultrafast laser pulse. A single-photon detector and a time-to-digital converter (TDC) are used to detect the fluorescence and time-tag the detected photons, respectively. Then a decay histogram is built, from which the lifetime is extracted [1]. Since FLIM is an indirect imaging technique, the data processing is a critical aspect. Traditionally to extract the fluorescence lifetimes, the measured fluorescence decay in each pixel needs to be fitted with single or multi-exponential decay models using nonlinear least-square fitting (NLSF) algorithms such as the Levenberg-Marquardt algorithm (LMA) and the trust-region-reflective algorithm (TRRA), or the maximum likelihood estimation (MLE) method. However, such iterative methods are computationally intensive and only suitable for off-line analysis. Prior knowledge of the lifetime parameters is also required for setting proper initial conditions. Furthermore, to obtain accurate fitting results with a high signal-to-noise ratio (SNR), it takes a long time to obtain a sufficient photon count. Some non-iterative fitting-free methods, such as the phasor analysis [8], [9] and the centre-of-mass method [10], [11] have been proposed to improve the analysis speed.

Recent advances in deep learning (DL) have profoundly impacted on various fields such as computer vision, robotics, natural language processing, and speed recognition [12]. DL features hierarchical representation learning, possessing a much more robust ability to learn complex functions and high dimensional data and outshining conventional machine-learning techniques. Convolutional neural networks (CNNs) have been the most extensively used architectures for DL. Compared with fully connected dense neural networks, CNNs show distinctive features of sparse interactions, parameter sharing, and equivariant representations, making them efficient with excellent feature extraction capabilities and insensitive to noise and distortion [13]. Various CNNs such as AlexNet, GoogLeNet, and Microsoft ResNet have achieved remarkable breakthroughs in machine learning [14]-[16]. DL is also a game-changer in biomedical fields. A wide range of exciting DL-based applications has been reported such as brain circuits reconstruction, predictions of drug molecules, super-resolution fluorescence microscopy, and analysis of three-dimensional fluorescence images [17]-[19]. DL also opens a door for fitting-free fast lifetime calculation in FLIM applications [20], [21]. Recently, a 3D CNN architecture, FLI-Net, has been proposed to rapidly analyze FLIM images 
with remarkable accuracy [22], [23]. Since CNNs are mainly designed for image analysis or speech processing, their architectures tend to be 2D, 3D, or even higher dimensions. The CNNs for fluorescence analysis also follow this principle, in which fluorescence data are treated as a 3D image with two spatial dimensions and one temporal dimension. CNNs can directly extract lifetime images without resorting to iterative fitting procedures.

Despite the successes of 2D or 3D CNNs for fluorescence lifetime analysis, we argue that only temporal decay features need to be evaluated for FLIM data analysis without considering spatial positions during the analysis. In other words, FLIM data should be treated as a 1D time series instead of a 3D matrix. It is straightforward because, in both point-scan and wide-field fluorescence lifetime measurements, the lifetime calculation process fits decay curves in a pixel-by-pixel manner. In some cases, spatial binning is required to improve the SNR, but the fitting process still works similarly. Further examining the 3D CNN architecture such as FLI-NET, we can find that the convolutional kernel size is $1 \times 1 \times N$ ( $N$ is an integer). The first two dimensions correspond to the spatial dimensions and the last one the temporal dimension [22]. It is shown that even in a higher dimensional $\mathrm{CNN}$, the neural network only extracts time series features while neglects spatial features. Applying high dimensional CNNs not only massively increases the training and calculation complexity, but also requires hardware with much more computing power and larger memory. 1D CNNs, on the other hand, show unparalleled advantages in many aspects. 1D CNNs have been widely used for real-time electrocardiogram monitoring and structural damage detection [24], [25]. The benefits of 1D CNNs are two-fold: 1) It is faster, as the complex matrix operations from high dimensional convolution are replaced by float-point additions and scalar multiplications in 1D CNNs. Thus, it is promising for real-time FLIM applications. The design and training of the neural network become easier as well. 2) It is easier to implement 1D CNNs on electronics hardware such as field-programmable gate array (FPGA) devices only requiring simple additions and multiplications [11], [26]. Simpler operations also guarantee simpler architectures and lower hardware resources required. Therefore, they are more hardware-friendly and suitable for cheap, portable devices with limited CPU computing power, memory, and power supplies. Besides, simple 1D CNN architectures allow processing multiple parallel series simultaneously, providing a fast and feasible way for processing FLIM images. They can work as embedded processors compatible with existing FLIM systems.

This paper will demonstrate the potential of 1D CNNs for FLIM applications by designing a 1D convolutional residual neural network (1D-ConvResNet). 1D-ConvResNet was developed to extract the lifetime parameters at a faster speed. We show that 1D-ConvResNet has a simpler architecture and therefore requires less training time. It can achieve the state-of-the-art performances of its 2D or 3D counterparts. The performances of our designed neural network have been quantitatively evaluated using synthetic fluorescence images. The developed neural network was also applied to analyzing

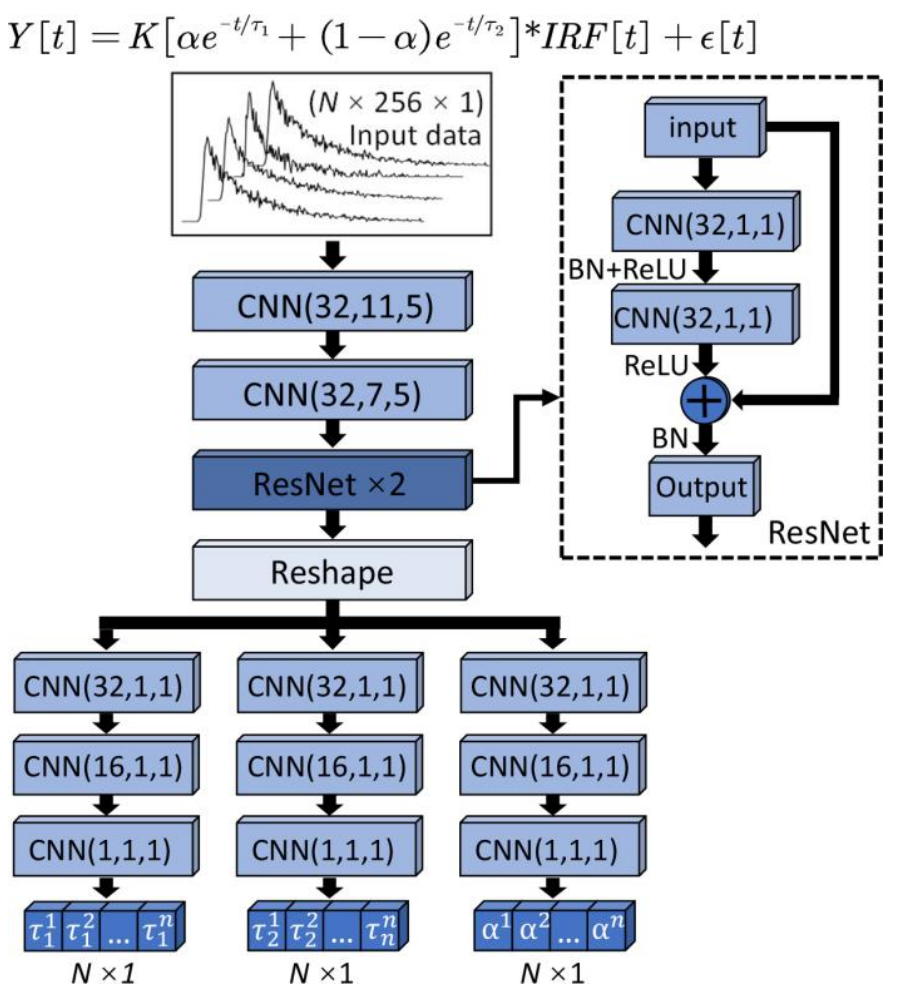

Fig. 1. The architecture of the proposed 1D ConvResNet for bi-exponential analysis. The detailed hyperparameters in each layer in parenthesis represent the neuron number, the kernel size, and the stride size, respectively. The input data is an $N \times 256 \times 1$ time series, where $N$ is an arbitrary integer. $Y[t]$ shows the mathematic model of the bi-exponential decays. The outputs are three $N \times 1$ arrays. The architecture of the ResNet block is shown in a dashed box. The batch normalization $(\mathrm{BN})$ layer and the nonlinear activation function rectified linear unit (ReLU) are added after the convolutional layers.

two-photon FLIM images of functionalized gold nanoprobes in Hek293 and human prostate cancer cells.

\section{ARCHITECTURE DESIGN}

Fig. 1 shows the topological structure of 1D ConvResNet for bi-exponential fluorescence decay analysis. The proposed neural network consists of two parts: the main branch for extracting the decay features and the sub-branches for reconstructing different parameters. For the network design, the first two CNN layers have a wider sliding window (larger kernel size) and a large stride so that they are expected to capture more general features of the decay curves. Following the CNN layers are two 1D residual neural networks (ResNet), through which the extracted features are learned by using residual learning [16]. Three output branches are designated for extracting three different lifetime parameters for bi-exponential decays (the short lifetime $\tau_{1}$, the long lifetime $\tau_{2}$, and the fraction ratio $\alpha$; see (A1) in Appendix), which are composed of three 1D CNN layers with pointwise convolution (kernel size = 1) and a decreasing neuron size. The purpose of using pointwise convolution is to implement feature pooling, thus effectively reconstructing the lifetime parameters in the final outputs. Two tricks were used to optimize our neural network: the ResNet and batch normalization (BN) [16], [27]. A ResNet is a network 
connection that adds a shortcut to reinject the past output to a later output. It aims to tackle two common problems: 1) the vanishing gradients and 2) representational bottlenecks during the network training [16]. The BN layer is included after each convolutional layer and before the activation function, which can adaptively normalize data to reduce the shift of internal covariance and accelerate network training [27]. The proposed 1D-ConvResNet was developed using TensorFlow2.0 [28]. The loss function of our designed neural network $L$ is:

$$
L(\Theta)=\frac{1}{N} \sum_{i=1}^{N} \sum_{j=1}^{M}\left\|F_{j}\left(Y^{i}, \Theta\right)-\hat{Y}_{j}^{i}\right\|_{2}^{2},
$$

where $F$ is $j^{\text {th }}(j=1, \ldots, M)$ end-to-end mapping function and $M$ is the number of the output branches $(M=3$ for bi-exponential decays). $Y$ is the input signal and $\hat{Y}_{j}$ is the corresponding ground-truth target of the $j^{\text {th }}$ output branch. $N$ is the batch size number. $\theta$ is the hyperparameter of the network, e.g., the trainable parameters of convolutional layers and BN layers. The activation function is rectified linear units (ReLU), and the optimizer used the root mean square propagation (RMSprop) algorithm with a learning rate of 1e-5 [13]. Other hyperparameters such as the kernel size and the stride of different convolutional layers were optimized based on the exhaustive searching method. The detailed hyperparameters of the network configuration are shown in Fig. 1.

The acquisition of the training data is critical for training a robust neural network. Since the mathematical model of the fluorescence decay curves and noise characteristics have been well developed, the training data can use synthetic decay curves with known parameters (see details in Appendix). The synthetic measured decay $Y[t]$ is the true decay $y[t]$ convolved with the instrument response function (IRF) plus noise:

$$
Y[t]=K \cdot \operatorname{IRF}[t]^{*} y[t]+\varepsilon[t]
$$

where $I R F[t]$ refers to the IRF function and $y[t]=\sum \alpha_{\mathrm{i}} e^{-\mathrm{t} /{ }_{i}}(i=1$, $2, \ldots, n)$ is the exponential decay model with $n$ lifetime components. The asterisk * denotes the continuous convolution operator in the time domain. In a TCSPC system, shot noise is dominant and therefore $\varepsilon(t)$ follows a Poisson distribution [7]. The signal-to-noise ratio (SNR) of the decay curves is the square root of the total photon counts:

$$
S N R=\sqrt{\sum_{t=1}^{N} Y[t]}
$$

where $N$ is the time bin number.

\section{NETWORK EVALUATION}

Fig. 2 shows the training and testing results of two different sample datasets: Datasets $A$ and $B$. Both training datasets contain 40000 decay curve samples, in which $20 \%$ of the
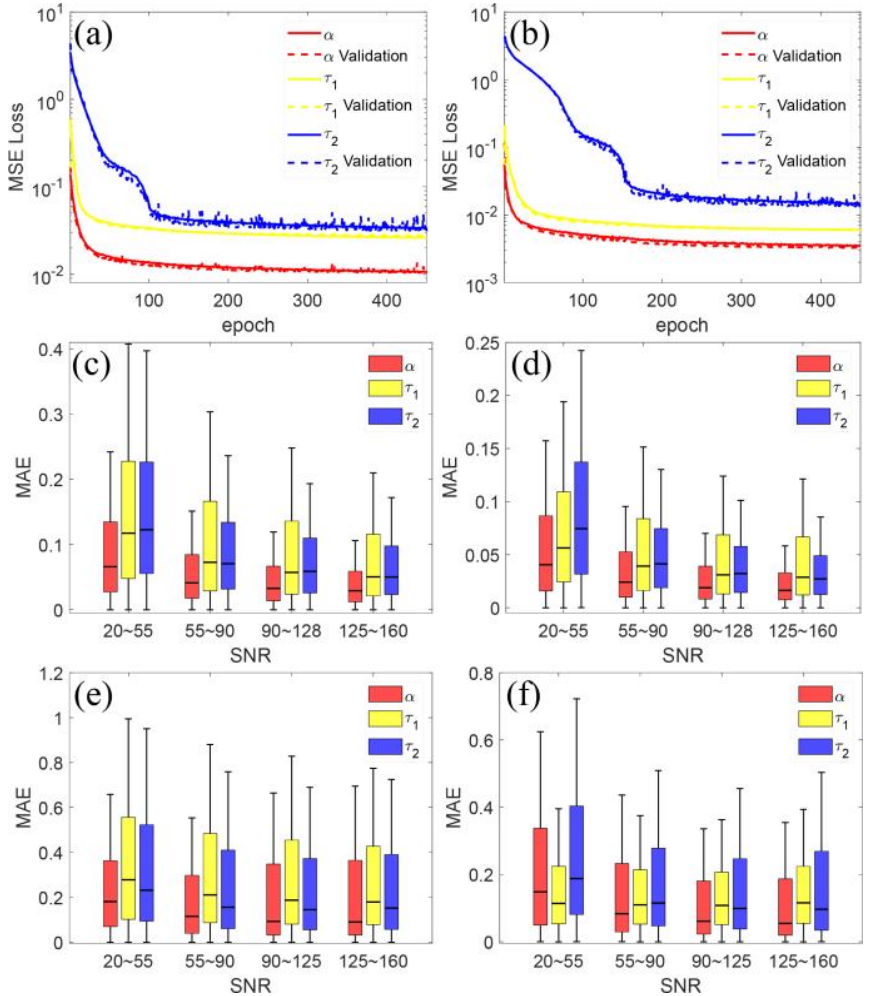

Fig. 2 (a) and (b), the mean absolute error (MAE) of the training and validation for $\tau_{1}, \tau_{2}$, and $\alpha$ using training Dataset $A$ (a) and $B$ (b), respectively. (c) and (d), the MAE of predicted results of the testing datasets for the three parameters when the network is trained by the Datasets $A$ (c) and $B(\mathrm{~d})$, respectively. (e) and (f), the corresponding fitting results of the testing datasets using TRRA when the network is trained by the Datasets $A(\mathrm{e})$ and $B(\mathrm{f})$, respectively.

samples are used as the validation subset. The batch size of the training is 128 and the training epochs is 500 . An early stop callback with 25 patient epochs (5\% of total epochs) is included to prevent overfitting. In Dataset $A, \tau_{1} \sim \mathrm{U}[0.1,1] \mathrm{ns}$ and $\tau_{2} \sim \mathrm{U}[1$, 3] ns. In Dataset $B, \tau_{l}$ has a relatively narrower range as $\tau_{l}$ $\sim \mathrm{U}[0.1,0.5] \mathrm{ns}$ whereas $\tau_{2}$ remains unchanged. Here $\mathrm{U}$ denotes uniform distribution. Our training lifetime range covers a wide range of commonly used fluorophores and fluorescent probes in biomedical applications. The SNR of the training datasets follows a uniform distribution as SNR U[20,160], covering a wider range of noise levels. Figs 2 (a) and (b) show the mean square error (MSE) loss of the training and validation data changing with the training epochs for Datasets $A$ and $B$, respectively. All three parameters converged at a minimal loss level. Compared with Figs 2 (a) and (b), the loss of $\tau_{1}$ is relatively large if trained by a dataset with a wider lifetime range. As a result, the predictions of $\tau_{l}$ would have large deviation. Figs 2 (c) shows the boxplot of the mean average error (MAE) of the predicted results of the testing datasets using the trained network as shown in Fig. 2(a). The testing dataset contains four datasets of samples with SNR ranging from 20 to160. Each dataset contains 4000 decay curves that were never seen by the neural network before. The lifetime range was kept the same as the training dataset. In Figs 2 (c), the mean MAEs of all three lifetime parameters decrease significantly as SNR increases, indicating more precise results. 
A noisier dataset (with a smaller SNR) has wider error distributions.

To improve lifetime extraction precision, one approach is to narrow the lifetime range of interest. It is applicable if we have prior knowledge of the lifetime distribution. As demonstrated in Fig. 2 (d), when $\tau_{l}$ is restricted to $0.1 \sim 0.5 \mathrm{~ns}$, the median MAE of $\tau_{l}$ and the corresponding error distribution range have smaller values in comparison with Fig. (c). The MAE distributions for $\tau_{2}$ and $\alpha$ are also confined within a smaller range for datasets with the SNR from 20 to 160. Further decreasing the lifetime ranges for $\tau_{1}$ and $\tau_{2}$ can obtain a lower MAE, showing that a narrower resolvable lifetime range could lead to higher prediction precision for all three parameters. For better comparison, the lifetime parameters of testing datasets in Fig. 2 (c) and (d) are also resolved using a traditional fitting algorithm. TRRA can solve a bound-constrained optimization problem (lsqcurvefit in MATLAB). The constrained bounds for $\tau_{1}, \tau_{2}$, and $\alpha$ were set the same with their ranges in the training datasets. Figs. (e) and (f) show the corresponding results for the testing datasets in Figs. (c) and (d), respectively. In the whole SNR range, the performance of TRRA is poorer than that of 1D-ConvResNet. The performance of 1D-ConvResNet for all lifetime parameters has notable improvement as the SNR increases. However, the performance of TRRA has no significant change when SNR varies from 55 to 160. This further demonstrates the robustness and accuracy of the 1D-ConvResNet. It is worth noting that the proposed neural network has an excellent ability to resolve the factional ratio $\alpha$ for a wide lifetime range. $\alpha$ plays a vital role in evaluating the energy transfer efficiency in FRET applications. In contrast, dense neural networks usually fail to extract this parameter [21]. Besides, no deconvolution is needed because the effect of the IRF on lifetime estimations is automatically considered. Although only one IRF is considered in the training data, 1D-ConvResNet shows a robust performance for IRF with a wide range of the full width at half maximum (FWHM) (Section B in Appendix).

The quantitative analysis of the MAE for $\tau_{1}$ and $\tau_{2}$ under different conditions was carried out to understand further the lifetime resolving capability of the 1D-ConvResNet. The neural network was trained using Dataset $B$. The new testing data have more comprehensive lifetime ranges with $\tau_{1}$ and $\tau_{2}$ in the range of $0.1-1 \mathrm{~ns}$ and $1-4 \mathrm{~ns}$, respectively, to investigate the lifetime resolving performance beyond the scope of training data. Fig. 3 shows the results for SNR ranging from 20 to 160 and $\alpha$ ranging from 0.1 to 0.9 . For both $\tau_{1}$ and $\tau_{2}$, MAE has a larger mean and standard deviation values for a smaller SNR, which agrees with the previous analysis. The resolving capability of the neural network highly depends on the training data. The neural network has a homogeneous resolving capability for the lifetimes within the range of the training data. However, the MAE of predicted results increases linearly when the lifetime exceeds the range of training data. Therefore, the resolving capability of the neural network is tunable according to the chosen training data. Also, $\alpha$ can affect the predicted results of $\tau_{1}$ and $\tau_{2}$. When a lifetime component has a predominant weight (for example, $\alpha \sim 1$ for $\tau_{l}$ ), it has better
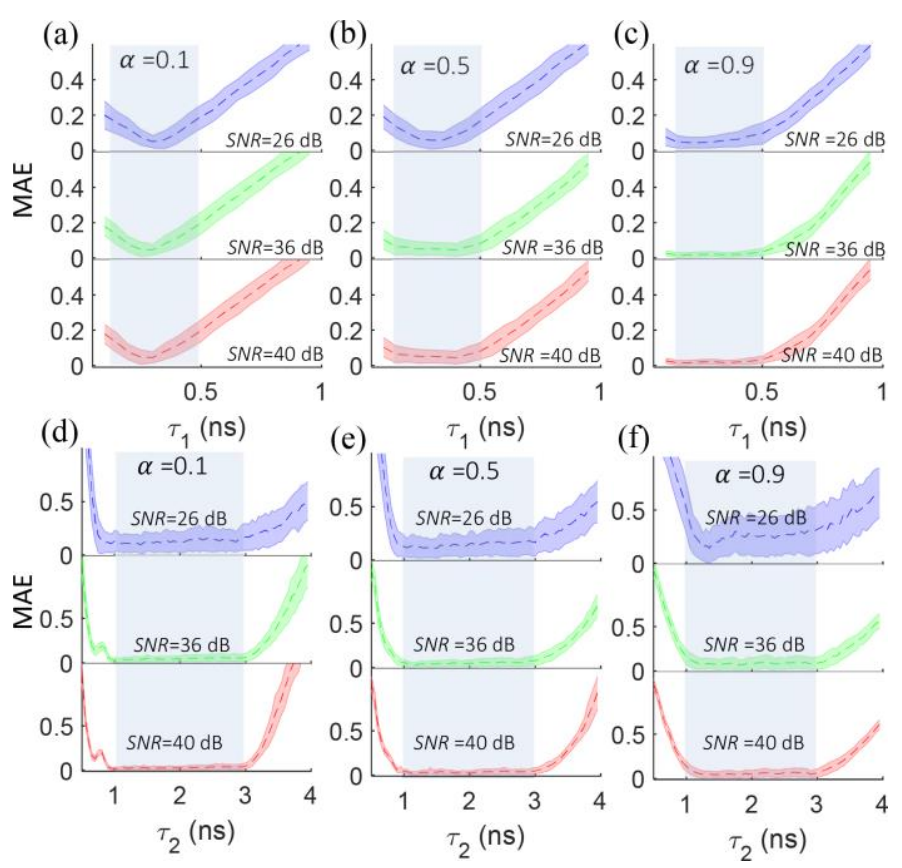

Fig. 3. The mean and deviation of MAE for $\tau_{l}$ (a)-(c) and $\tau_{2}$ (d)-(f) under different conditions. SNR takes the values of 20,60, and 100; $\alpha$ takes the values of $0.1,0.5$, and 0.9 . In the analysis, $\tau_{2}$ is fixed at $2.5 \mathrm{~ns}$ in (a) - (c) and $\tau_{l}$ is fixed at $0.3 \mathrm{~ns}$ in (d) - (f). The dashed areas denote the lifetime ranges of training data. In each subplot, the mean and standard deviation of the MAE of 50 random-generated decays are represented by a dashed line and shaded area, respectively.

precision whereas others have lower precision.

The proposed 1D-ConvResNet can be easily modified for $p$-exponential decay models ( $p$ is the number of lifetime components) by having $2 p-1$ sub-branches without redesigning the $\mathrm{CNN}$ architecture. Fig. 4 shows an example for resolving a tri-exponential decay, i.e., $p=3$, with three different lifetime components $\tau_{i}(i=1,2,3)$, two independent fraction ratio $\alpha_{\mathrm{i}}(i=1,2)$, and $\alpha_{3}=1-\alpha_{1}-\alpha_{2}$. Shown in Fig. 4 (a), each parameter is assigned to a branch. The hyperparameters of the main branch and sub-branches remain the same as Fig. 1. The training dataset contains 40,000 different samples. The parameter ranges of the training dataset are: $\tau_{1} \sim \mathrm{U}[0.1,0.4]$, $\tau_{2} \sim \mathrm{U}[0.9,1.2]$, and $\tau_{3} \sim \mathrm{U}[1.8,2.2]$. $\alpha_{1}, \alpha_{2}$, and $\alpha_{3}$ obey a uniform marginal distribution in the range from 0 to 1 and subject to $\alpha_{1}+$ $\alpha_{2}+\alpha_{3}=1$. Fig. 4 (b) shows the training and validation MAE for the five parameters. All of them are well converged at MSEs below 0.01 after 200 epochs. The testing results of new data with the same ranges as the training data are shown in Fig. 4 (c). Astonishingly, the 1D-ConvResNet has high prediction precision for all parameters with SNR ranging from $20 \sim 160$. In contrast, in Fig. 4(d), TRRA delivers poor results for all parameters, especially the $\alpha_{1}$ and $\alpha_{2}$. The performance of TRRA nearly keeps unchanged within the whole SNR range.

We further quantitatively investigate our proposed neural network's gains in terms of the precisions of different parameters and the computational speed compared with TRRA. Since the performances of both CNN and TRRA have a large variation for resolving fluorescence decays with different lifetime parameter combinations and SNR, it is meaningless to compare their performances case-by-case. Here the 

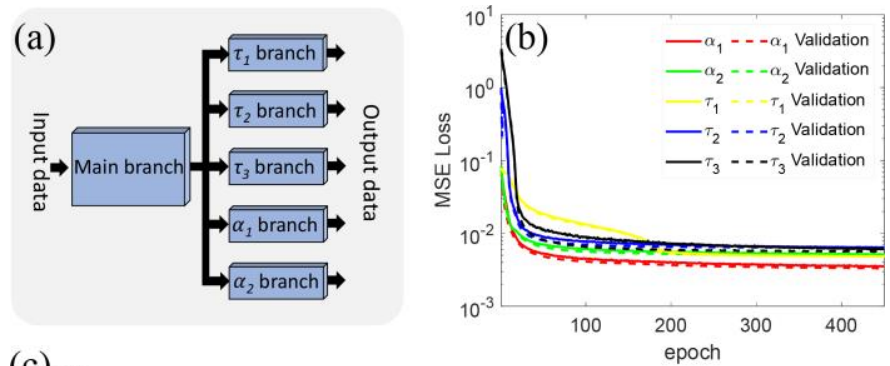

$(\mathrm{c})_{0.25}$

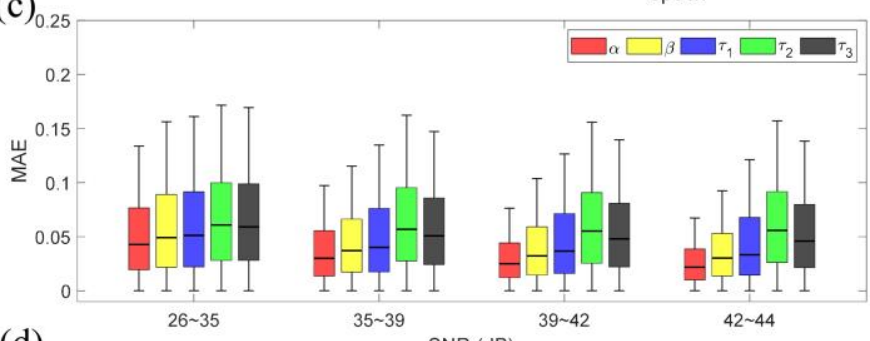

(d)

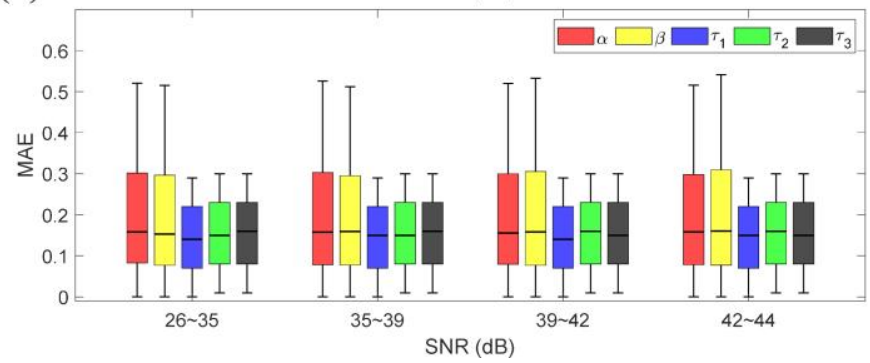

Fig. 4 (a) The modification of 1D-ConvResNet for tri-exponential decay model analysis. (b) The MSE loss of training and validation for a testing dataset with tri-exponential decays. The SNR of the testing dataset is from $26 \mathrm{~dB}$ to $44 \mathrm{~dB}$. (c) and (d), The corresponding predicted results for $\tau_{\mathrm{i}}(i=1,2,3)$ and $\alpha_{i}(i=1,2)$ of new testing samples using 1D-ConvResNet and TRRA, respectively.

figure-of-merit (F-value) was introduced as an indicator to compare the overall precision of different algorithms. F-value is defined as [29]:

$$
F=\frac{\delta x}{x} S N R,
$$

where $x$ is lifetime parameters $\left(\tau_{\mathrm{i}}\right.$ or $\left.\alpha_{i}\right)$ and $\delta x$ is the standard deviation of the lifetime parameter determination. $F$ is a normalized value for measuring the sensitivity of the lifetime parameter acquisition algorithm. A low value of $F$ indicates a high precision. Hence, the precision gain can be simply defined as $G_{x}=\left[F_{x}^{N L S D} / F_{x}^{C N N}\right]^{2}$ [29]. Table I summarized the fitting/prediction performance and calculation speed comparison for bi- and tri- exponential decay models. The F-values are obtained from the testing datasets for the bi- and tri- exponential decay model with 40,000 samples in Figs. 2(d) and 4(c). It is demonstrated that our proposed CNN algorithm significantly outperforms traditional TRRA with large precision gains. Besides, in comparison with TRRA, CNN shows a 300 -fold increase in average calculation speed under the same hardware platform (Intel i7-4790 CPU).
TABLE I

FITTING PERFORMANCE COMPARISON

\begin{tabular}{|c|c|c|c|c|c|}
\hline \multicolumn{6}{|c|}{ bi-exponential decay model } \\
\hline Parameter & $\tau_{l}$ & & $\tau_{2}$ & & $\alpha$ \\
\hline$F(\mathrm{CNN})$ & 35.5 & & 5.0 & & 79.8 \\
\hline$F$ (TRRA) & 82.6 & & 19.7 & & 640 \\
\hline Precision Gain & 5.3 & & 15.2 & & 25.0 \\
\hline \multicolumn{6}{|c|}{ tri-exponential decay model } \\
\hline Parameter & $\tau_{l}$ & $\tau_{2}$ & $\tau_{3}$ & $\alpha_{1}$ & $\alpha_{2}$ \\
\hline$F(\mathrm{CNN})$ & 35.1 & 7.5 & 3.7 & 905.5 & $1.75 \mathrm{e} 3$ \\
\hline$F($ TRRA $)$ & 403 & 44.3 & 36.3 & $6.7 \mathrm{e} 3$ & $4.2 \mathrm{e} 3$ \\
\hline Precision Gain & 132.3 & 34.8 & 96.0 & 54.8 & 5.8 \\
\hline \multicolumn{6}{|c|}{ average speed ( $\mathrm{ms} / \mathrm{sample})$} \\
\hline $\mathrm{CNN}$ & \multicolumn{5}{|c|}{0.12} \\
\hline TRRA & \multicolumn{5}{|c|}{37.5} \\
\hline Speed Increase & \multicolumn{5}{|c|}{$\approx \mathbf{3 0 0}$} \\
\hline
\end{tabular}

Unlike the training of 2D or 3D CNNs that usually relies on high-performance GPUs with a massive number of parallel processors or cloud computing, the proposed 1D-ConvResNet for both bi-, tri-, or even multi-exponential decay models can be performed much more quickly with desktop computers. This is due to the efficient neural network architecture. Table II summarized the architecture comparison with previously reported high dimensional CNN and feed-forward dense neural network. The total parameter is only about $4.5 \%$ of the $3 \mathrm{D}$ FLINET, indicating that a large memory and floating operation can be saved. The whole training procedure only took tens of minutes on a laptop with the Intel i7-4790 CPU. In contrast, higher-dimensional CNN architectures usually take several hours even with GPU acceleration [22], [23]. Shorter training time is beneficial because it ensures that CNN can be rapidly re-trained and deployed for different FLIM applications.

TABLE II

ARCHITECTURE COMPARISON

\begin{tabular}{cccc}
\hline \hline & 1D-ConvResNet & $\begin{array}{c}\text { FLINET } \\
{[22]}\end{array}$ & $\begin{array}{c}\text { DENSE } \\
\text { NET } \\
{[21]}\end{array}$ \\
\hline $\begin{array}{c}\text { Total Parameters } \\
\text { Resolving }\end{array}$ & $\mathbf{4 8 , 6 7 5}$ & $1,084,045$ & 149,252 \\
$\begin{array}{c}\text { multi-exponential } \\
\text { decay models? }\end{array}$ & Yes & Yes & No \\
$\begin{array}{c}\text { Training platform } \\
\text { Training time }\end{array}(h)$ & CPU & GPU & CPU \\
\hline \hline
\end{tabular}

${ }^{a}$ For a fair comparison, the time bin is set to be 256 for all three networks. The neuron number of the 4-layer DENSE NET is set as $256,256,256$, and 4, since the author did not show explicit neuron numbers in [21].

${ }^{b}$ The training processor is Intel i7-4790 CPU for our network NVIDIA TITAN Xp GPU for [22], and Intel(R) Xeon(R) E31245 for [21]

Compared with $2 \mathrm{D}$ or $3 \mathrm{D}$ CNNs, $1 \mathrm{D}-\mathrm{CNNs}$ are more suitable and flexible for analyzing fluorescence images as a hardware-embedded processor. In higher dimensional CNNs, the neural network should process all pixels in a fluorescence image at once. However, in many applications, a fluorescence 


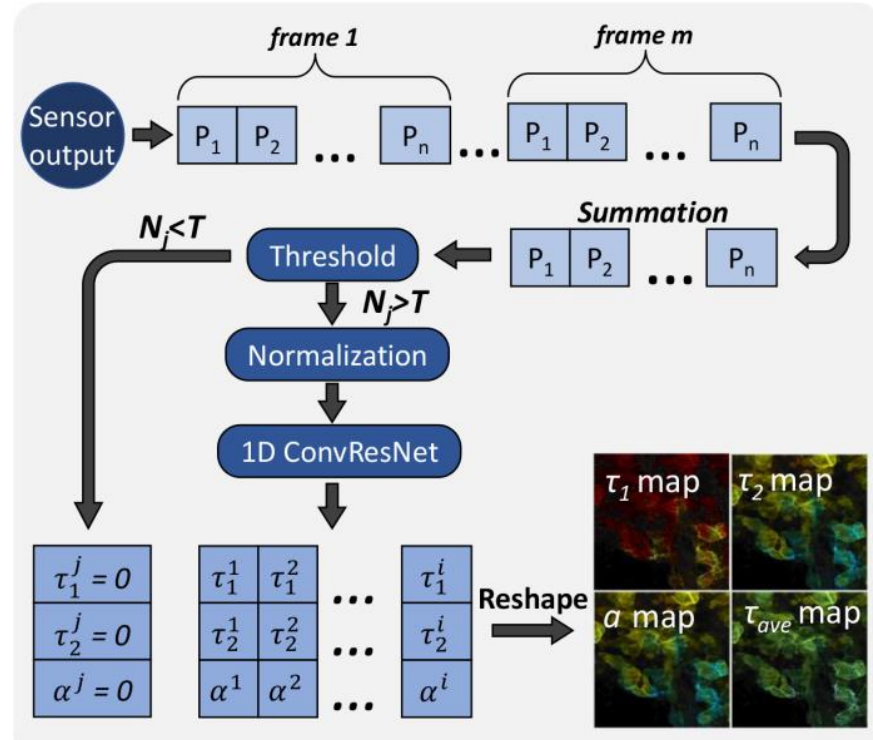

Fig. 5. Workflow of 1D-ConvResNet for calculating 2D FLIM images obtained from a sensor. The data readout of the sensor follows the pipeline principle. The raw output data flow is arranged pixel-by-pixel and frame-by-frame. $\mathrm{P}_{\mathrm{i}}$ refers to the pixel in the sensor. 1D CNN can directly work as an embedded processor to process the data.

image has a sparse feature that contains a large background area with no useful information. The pixels in this area are also included in such a neural network to produce reconstructed lifetime images, leading to low calculation efficiency. In contrast, 1D CNNs have multiple parallel series processing capabilities that allow arbitrary pixels to be processed simultaneously (see Fig. 1). It is easy to set a threshold or choose regions of interest for faster analysis. More importantly, the data readout of sensors such as photomultipliers (PMTs) and single-photon avalanche diodes (SPADs) in FLIM systems usually follows a pipeline principle, i.e., the measured raw data is sorted pixel-by-pixel and frame-by-frame. 1D CNNs can directly process the output data without extra data conversion. The image processing procedure is shown in Fig. 5. The embedded CNN processor can focus on the pixels with intensities above the threshold. Those below the threshold are considered as background pixels, and their lifetimes are ignored.

\section{EXPERIMENTAL RESULTS AND DISCUSSIONS}

The proposed neural network was further employed to analyze two-photon FLIM images of functionalized gold nanoprobes in living cells. Gold nanorods (GNRs) support shape-dependent localized surface plasmon resonance, leading to tunable optical properties and strong two-photon luminescence [30]. GNRs are efficient and stable fluorescence quenchers for organic fluorophores [31]. Gold nanoprobes are GNRs functionalized by fluorophore-labelled hairpin oligonucleotides, which have found various biomedical applications including RNA detection for cancer diagnosis, research of energy transfer behaviours, and recognition of analytes [32], [33]. In this study, GNRs were functionalized by Cy5 labelled thiolated single-strand DNAs (Cy5-ssDNA-GNRs)
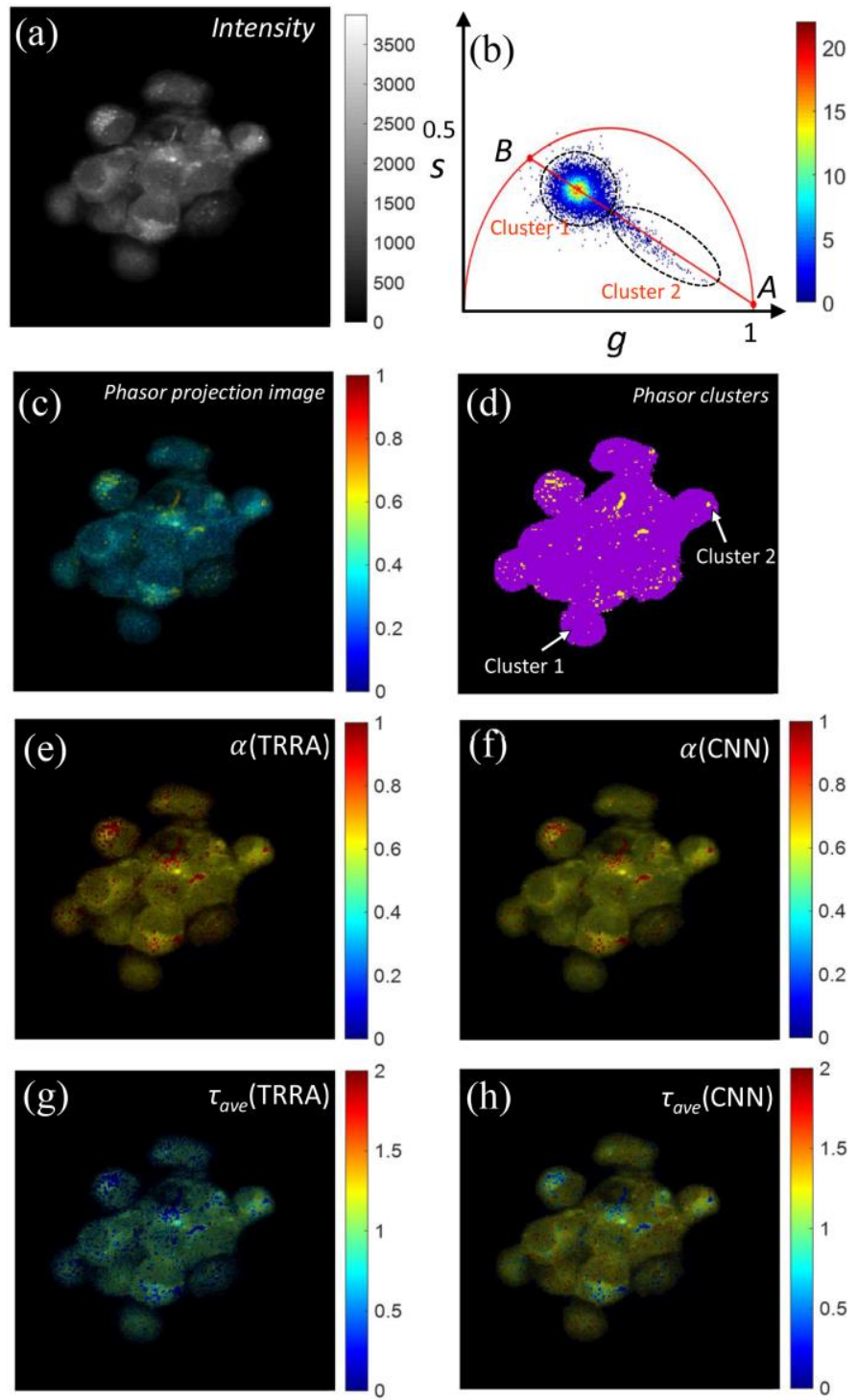

Fig. 6. FLIM images of Cy5-ssDNA-GNRs labeled Hek293 cells. (a) Intensity image; (b) phasor plot, the red line is the fitted line of the phasor pixels. A threshold with $20 \%$ the maximum photon counts is set for fitting the line. $A$ and $B$ are the intersection points between the fitted line and the semicircle. Two dashed ellipses indicate two clusters; (c)-(d) the phasor projection cluster maps; Cluster 1 and 2 denote the cell and gold nanoprobes, respectively. (e)-(f) $\alpha$ maps and (g)-(h) $\tau_{\text {ave }}$ maps calculated by TRRA and CNN.

via ligand exchange and salt aging processes [32]. To identify the locations of endocytic gold nanoprobes in living cells, FLIM images of Cy5-ssDNA-GNRs labelled Hek293 cells and human prostatic cancer cells were evaluated. Briefly, the cells were treated with gold nanoprobes solution for incubation; then, the samples were washed with phosphate-buffered saline (PBS) and fixed with paraformaldehyde. The sample was dispersed on a glass slide after staining with 4'-6-diamidino-2-phenylindole (DAPI), then the coverslips were mounted for microscopy with a ProLong antifade solution. FLIM images were acquired by a two-photon FLIM system. It includes a confocal microscope (LSM 510, Carl Zeiss, Oberkochen, Germany), a femtosecond Ti: sapphire laser (Chameleon, Coherent, Santa Clara, USA), and a TCSPC acquisition system (SPC-830, Becker \& Hickl $\mathrm{GmbH}$, Berlin, Germany). The laser excitation wavelength is 
$850 \mathrm{~nm}$, and the laser pulse width is less than $200 \mathrm{fs}$ with an $80 \mathrm{MHz}$ repetition rate. The bin width of the TCSPC is $0.039 \mathrm{~ns}$, and each measured histogram contains 256 -time bins. The emission light was collected by a $60 \times$ water-immersion objective lens (N.A $=1.0$ ) with a 500-550 nm bandpass filter.

The first case is the FLIM image of Cy5-ssDNA-GNRs labelled Hek293 cells. Fig. 6 (a) shows the gray-scale fluorescence intensity image. Compared with cell tissues, gold nanoprobes show higher intensities due to their strong two-photon luminescence. However, it is difficult to precisely identify their locations solely from intensity images because the amount of gold nanoprobes is relatively small. The fluorescence intensities of cells have a large variance. The two-photon luminescence of GNR has ultra-short lifetimes around tens of picoseconds [34]. The free state Cy5 has a lifetime around $2 \mathrm{~ns}$. When Cy5 is bounded in a closed hairpin oligonucleotide structure attached to a gold surface, Cy5 is in a quenched state. It has a short lifetime in the sub-nanosecond range because of the intense energy transfer between Cy5 and GNRs. Hence, gold nanoprobes show complex ultra-short lifetimes. Fig. 6 (b) shows the corresponding phasor plot of the FLIM image. A threshold of 100 is set to filter out low-intensity pixels. Two clusters can be observed from the phasor plot. Cluster 1 with majority pixels is the primary cluster located near $B$, showing that these pixels have longer lifetimes. Another cluster with a tail extending to the right bottom can also be found. The number of pixels in Cluster 2 is smaller, and the lifetimes are shorter. The whole phasor pixels nearly show a straight-line distribution, suggesting that a bi-exponential decay model can analyze the FLIM image. Before conducting CNN or TRRA analysis, the phasor orthogonal projection and cluster images of the FLIM data were calculated (see details in Appendix). Since there is no ground truth for experimental data, the phasor and cluster images can serve as a reference for comparing the TRRA and CNN results. Fig.6 (c) shows the pseudo-colour image of the phasor projection score $\beta$, in which each phasor pixel is projected on the line segment between $A$ and $B$ with $\beta$ being the relative distance from point $B$. The two points $A$ and $B$ are the intersection points between the fitted line for the pixels and semicircle. $\beta$ changes from 0 to 1 as the projected position shifts from $B$ to $A$. When the projected position is out of the line segment, $\beta$ is set to 0 .

It should be noted here that $\beta$ has a different meaning to $\alpha$ because the pixels in the phasor plot follow a nonlinear spatial distribution from Fourier transform. Nevertheless, $\beta$ is sufficient to distinguish gold nanoprobes from cell tissues in Fig. 6 (c). Meanwhile, $\beta$ can also be used as a criterion to segment Clusters 1 and 2. Fig. 6 (d) shows the corresponding cluster image for $\beta=0.4$; the pixels in Cluster 1 are attributed to the autofluorescence of cells, whereas Cluster 2 are attributed to the endocytic gold nanoprobes. Figs 6 (e)-(f) and (g)-(h) show the calculated $\alpha$ maps and amplitude-weighted average lifetime $\left(\tau_{\text {ave }}\right)$ maps using TRRA and CNN, respectively. The intensity threshold is 100 photon counts. Since the pixels of nanoprobes should have a larger $\alpha$ value, the red pixels in Figs 6 (e) and (f) indicate the gold nanoprobes' locations. Compared with the results in Fig. 6 (d), both TRRA and CNN can obtain
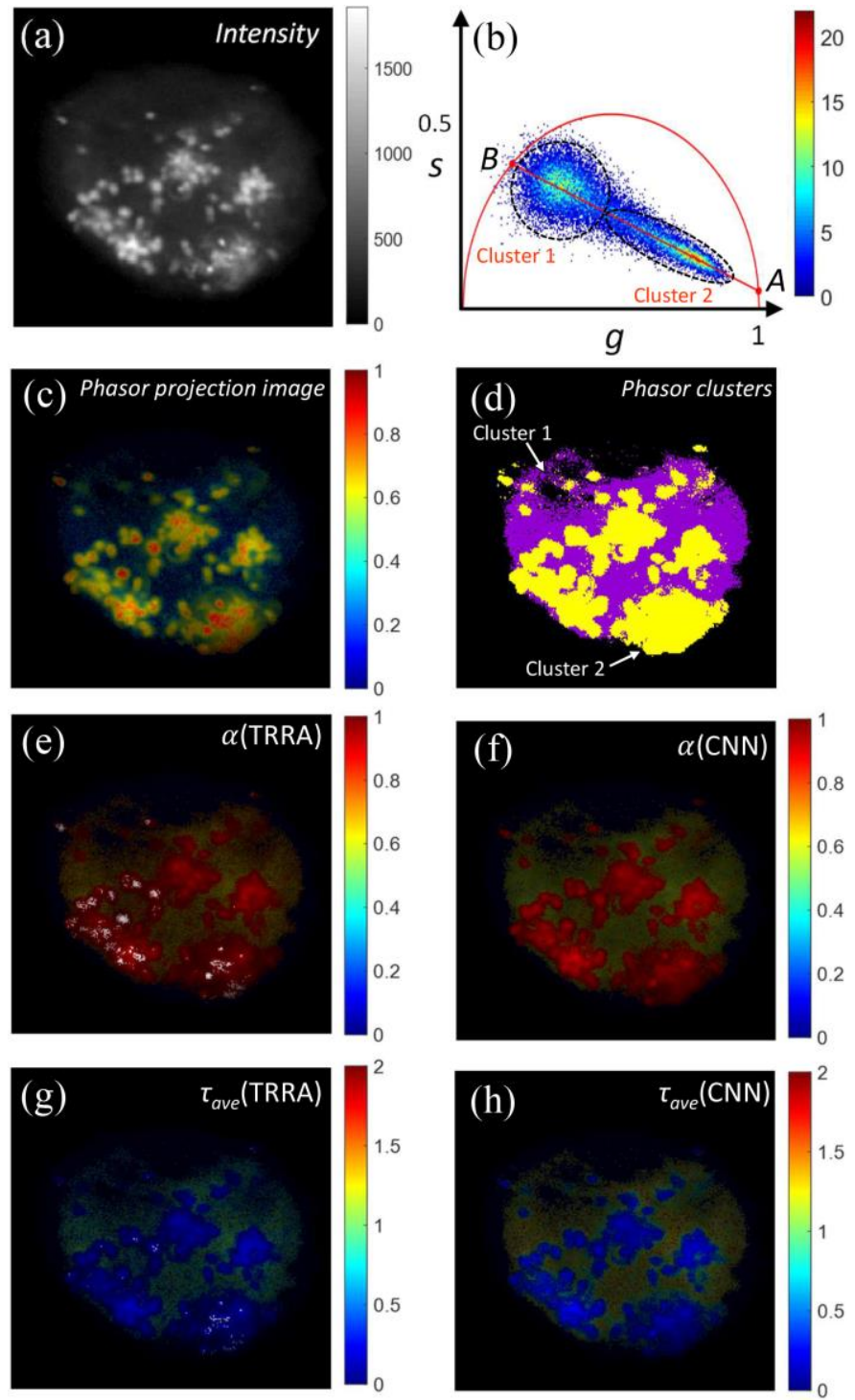

Fig. 7. FLIM images of a single human prostatic cancer cell with endocytic Cy5-ssDNA-GNRs. (a) Intensity image; (b) phasor plot. (c)-(d) phasor projection and cluster maps; Cluster 1 and 2 denote the cell and gold nanoprobes, respectively. White pixels mean that $\alpha$ is beyond [0,1]; (e)-(f) $\alpha$ maps and (g)-(h) $\tau_{\text {ave }}$ maps obtained by TRRA and CNN.

similar conclusions about the gold nanoprobes' locations to the phasor cluster analysis. However, CNN's $\alpha$ map can more accurately identify gold nanoprobes, whereas TRRA -estimated images are noisier due to a more extensive deviation range. It becomes worse for TRRA to resolve $\tau_{\text {ave }}$. Fig. 6 (g) shows the $\tau_{\text {ave }}$ map calculated by TRRA; the blue pixels with shorter lifetimes indicate the gold nanoprobes' locations. It is difficult to identify gold nanoprobes form Fig. $6(\mathrm{~g})$, suggesting that TRRA has worse performance in resolving $\tau_{\text {ave }}$. In contrast, $\mathrm{CNN}$-estimated $\tau_{\text {ave }}$ and $\alpha$ maps agree with the phasor cluster analysis, locating gold nanoprobes more accurately.

The second case shown in Fig. 7 is the FLIM image of a single human prostatic cancer cell with endocytic Cy5-ssDNA-GNRs. Unlike Hek293 cells in Fig. 6, the average intensity is lower and there are abundant endocytic gold nanoprobes. The intensities of gold nanoprobes are generally higher than 1000 similar to the first case. In contrast, only a few 
hundred average photon counts were collected from the cell in the second case. Fig. 7 (b) shows the corresponding phasor plot. The threshold is set to 100 counts. The pixel number in Cluster 2 shows a significant increase, attributed to an increased number of endocytic gold nanoprobes. Besides, the pixel distribution spreads to a broader range in Cluster 1 due to a low SNR level. Again, the phasor projection and cluster maps are shown in Fig. 7 (c) and (d), respectively. $\beta$ is set to 0.4 to segment the two clusters, the gold nanoprobes and the cells. The TRRA - and CNN-estimated $\alpha$ maps are shown in Fig. 7 (e) and (f), respectively. However, TRRA fails to find converged estimations in many pixels (white pixel areas) in Fig. 7(e). These pixels correspond to higher concentrated gold nanoprobes and have ultra-short decays, leading to divergent solutions in the deconvolution procedure. The TRRA -estimated $\tau_{\text {ave }}$ map shown in Fig. 7 (g) also fails to identify gold nanoprobes robustly. Instead, CNN provides more precise $\tau_{\text {ave }}$ map in Fig. 7 (h), more accurately identifying gold nanoprobes. The autofluorescence background in the cell was also resolved compared with Fig. 7 (g). It is shown that $\mathrm{CNN}$ is more robust under low count conditions.

\section{CONCLUSION}

In summary, we present the design and training of 1D ConvResNet for FLIM data analysis. The proposed method is more straightforward, requires fewer hardware resources, and is faster than traditional 2D/3D approaches. 1D ConvResNet can be easily trained using synthetic FLIM data for different FLIM applications. The simulated FLIM data analysis shows that CNN has superior performances in analyzing FLIM images with a wide range of intensity levels. The trained $\mathrm{CNN}$ was then employed to analyze two-photon FLIM images of functionalized gold nanoprobes in Hek293 and human prostate cancer cells. The results also suggest CNN's superior performances and robustness. Currently, the calculation time of the proposed 1D ConvResNet architecture for a $256 \times 256$ image takes several seconds on a laptop for a quick proof-of-concept demonstration. However, it can be further optimized using quantized neural network architectures and can also be implemented in FPGA devices to accelerate the analysis for video-rate FLIM applications. 1D ConvResNet is promising for a broader range of FLIM applications.

\section{ACKNOWLEDGEMENT}

This work is supported in part by the Medical Research Scotland (MRS-1179-2017), Photon Force, Ltd., Edinburgh, and the Biotechnology and Biological Sciences Research Council (BB/K013416/1).

\section{APPENDIX}

\section{A. Preparation of training data}

The simulated FLIM datasets for training the neural network were generated using home-made code in MATLAB
(MathWorks Inc., Massachusetts, USA). In our simulation, the number of time bins $N$ is set as 256 . The bin width $h$ is $0.039 \mathrm{~ns}$ and the observation period $T$ is $10 \mathrm{~ns}$. One can specify the simulation parameters according to different FLIM systems. The multi-exponential decay mode is described by:

$$
Y[t]=K\left[\sum_{i=1}^{n} \alpha_{i} e^{-t / \tau_{i}}\right] * I R F[t]+\varepsilon[t], t=1, \ldots, N
$$

where the fraction ratio $\alpha_{i}(i=1, \ldots, n)$ obeys a uniform marginal distribution in the range from 0 to 1 with a fixed sum $1 . \operatorname{IRF}(t)$ is fitted with a Gaussian function and expressed as $\operatorname{IRF}[t]=e^{\left[-\left(t-t_{0}\right)^{2} \cdot 4 \ln 2 h^{2} / F W H M^{2}\right]}$, where $t_{0}$ is the peak position. To mimic the laser jitter, $t_{0}$ has a uniform distribution within $13-15^{\text {th }}$ time bin. The FWHM is 167.3 ps. The convolution result of the decay curves and $\operatorname{IRF}(t)$ was normalized to 1 and then multiplied by peak intensity $K$ (maximum photon counts). $\varepsilon(t)$ is the Poisson noise. For larger photon counts (generally $>20), \varepsilon(t)$ can be approximated by a normal distribution and expressed as:

$$
\varepsilon[t]=\sqrt{K\left[\sum_{i=1}^{n} \alpha_{i} e^{-t / \tau_{i}}\right] * \operatorname{IRF}[t]} \cdot \mathscr{N}(0,1),
$$

where $\mathscr{N}(0,1)$ represents a normal distribution with zero mean and standard deviation of 1 . To generate specified $S N R$, the peak intensity $K$ can be set as

$$
K=S N R^{2} / \sum_{t=1}^{N}\left[\left[\sum_{i=1}^{n} \alpha_{i} e^{-t / \tau_{i}}\right] * \operatorname{IRF}[t]\right]
$$

Fig. A1 shows examples of generated bi- and tri-exponential decays with different peak intensities.
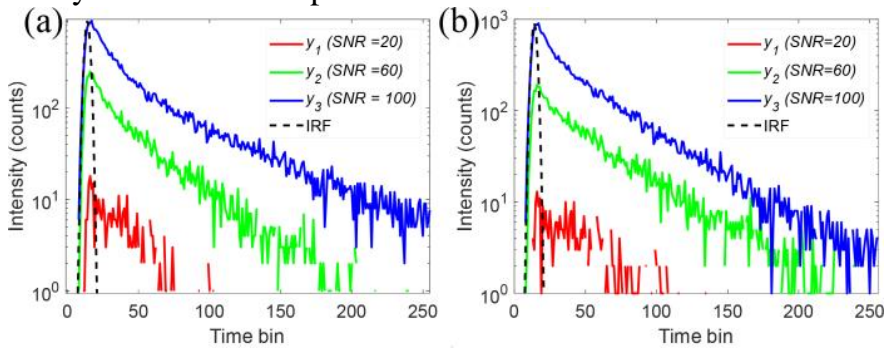

Fig. A1 (a) Examples of simulated bi-exponential decay curves with different peak intensities. The parameters $\left(\tau_{1}, \tau_{2}, \alpha, S N R\right)$ of $y 1, y 2$, and $y 3$ are $(0.4,2.8,0.6,20),(0.3,2.3,0.5,60)$, and $(0.3,2,0.7,100)$, respectively. The dashed line represents the Gaussian IRF. (b) Examples of simulated tri-exponential decay curves with different peak intensities. The parameters $\left(\tau_{1}, \tau_{2}, \tau_{3}, \alpha_{1}, \alpha_{2}, S N R\right)$ of $y 1, y 2$, and $y 3$ are $(0.2,0.9,1.8,0.2,0.3,20),(0.2$, $1.1,2.0,0.2,0.6,60)$, and $(0.1,1.1,2.0,0.6,0.3,100)$, respectively. The dashed line represents the Gaussian IRF.

\section{B. FWHM of the IRF}

Fig. A2 further investigates how the FWHM of the IRF affects the training and prediction of the neural network. A new dataset was generated with the same parameters as Dataset $B$ in the main context. But the FWHM of the convolved IRF in each decay sample was randomly selected from 100 300 ps. This 
FWHM range is typical in most laboratory FLIM systems. In Fig. A2 (a), the MAE curves of all three parameters are well converged at slightly higher values than those in Fig. 2. But the predicted results for test datasets are nearly the same as Fig. 2. It shows that the designed 1D ConvResNet is robust for varied IRFs.
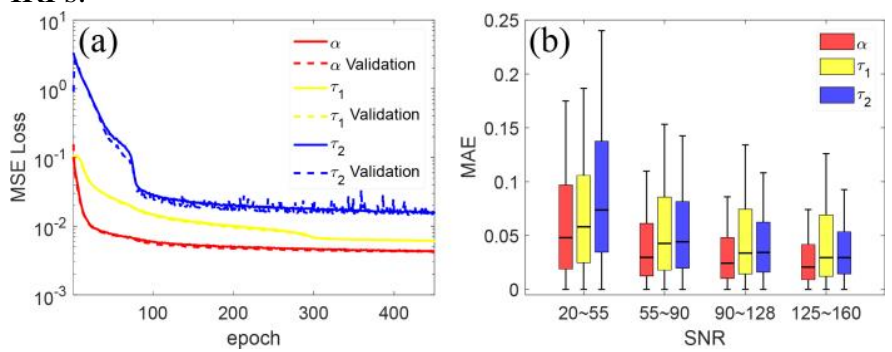

Fig. A2 (a) MSE loss of the training and validation for $\tau_{1}, \tau_{2}$, and $\alpha$ using the training dataset with $\tau_{1}$ and $\tau_{2}$ in the range of $0.1 \sim 0.5$ and $1 \sim 3 \mathrm{~ns}$. (b) Predicted results using the test dataset for $\tau_{1}, \tau_{2}$, and $\alpha$, respectively. The lifetime parameters are $\tau_{1} \sim \mathrm{U}[0.1,0.5] \mathrm{ns}, \tau_{2} \sim \mathrm{U}[1,3] \mathrm{ns}$, and $\alpha \sim \mathrm{U}[0,1]$.

\section{Phasor analysis}

The phasor analysis is to transform the FLIM data in the temporal domain to the phasor domain ( $g$-s-plane) through Fourier transform [8]:

$$
\left\{\begin{array}{l}
g_{i, j}(\omega)=\int_{0}^{\infty} Y_{i, j}(t) \cos (k \omega t) d t / \int_{0}^{\infty} Y_{i, j}(t) d t \\
s_{i, j}(\omega)=\int_{0}^{\infty} Y_{i, j}(t) \sin (k \omega t) d t / \int_{0}^{\infty} Y_{i, j}(t) d t
\end{array}\right.
$$

where $i, j$ are the coordinate indices of a FLIM image. The phasor analysis was performed using a home-made code written in MATLAB. Each pixel with the intensity over the predefined threshold was transformed to the phasor plane. The angular frequency is $k \omega=2 \pi / T$. Besides, the effect of IRF distortion is also taken into consideration. The convolution of IRF in the temporal domain is equivalent to the rotation of phasor points in the phasor plane. The calibration of IRF in the phasor plane is expressed as:

$$
\left[\begin{array}{l}
g_{i, j}^{c}(\omega) \\
s_{i, j}^{c}(w)
\end{array}\right]=\left[\begin{array}{cc}
g^{I R F}(\omega) & -s^{I R F}(\omega) \\
s^{I R F}(\omega) & g^{I R F}(\omega)
\end{array}\right] \cdot\left[\begin{array}{l}
g_{i, j}(\omega) \\
s_{i, j}(w)
\end{array}\right]
$$

where $g^{c}(\omega)$ and $s^{c}(\omega)$ are the calibrated phasor coordinates. $g^{I R F}(\omega)$ and $s^{I R F}(\omega)$ are the real and imaginary parts of the Fourier transform of the IRF.

For the phasor orthogonal projection analysis, firstly, a linear model $s=a g+b$ is used to fit the phasor points $\left(g_{n}, s_{\mathrm{n}}\right)$ :

$$
\underset{a, b}{\operatorname{minimize}} \sum_{n=1}^{N}\left\|s_{n}-\left(a g_{n}+b\right)\right\|_{2}^{2},
$$

where $a, b$ are the slope and intercept of the linear model, $N$ is the total phasor points. It is worth noting that a threshold in the phasor plot is critical for the linear fitting because a massive number of pixels with low photon counts would heavily bias the result, especially for low-SNR conditions. The coordinates of the two-intersection points $A(\mathrm{G} 1, \mathrm{~S} 1)$ and $B(\mathrm{G} 2, \mathrm{~S} 2)$ can be derived as:

$$
\left\{\begin{array}{l}
G 1=\frac{1-2 a b+\sqrt{(2 a b-1)^{2}-4\left(1+a^{2}\right) b^{2}}}{2\left(1+a^{2}\right)} \\
S 1=a G 1+b \\
G 2=\frac{1-2 a b-\sqrt{(2 a b-1)^{2}-4\left(1+a^{2}\right) b^{2}}}{2\left(1+a^{2}\right)} \\
S 2=a G 2+b
\end{array}\right.
$$

When the orthogonal projection of the phasor point is within the $A B$ segment, a projection score $\beta$ can be defined as:

$$
\beta_{n}=\left[\left(g_{n}-G 2\right)(G 1-G 2)+\left(s_{n}-S 2\right)(S 1-S 2)\right] / L,
$$

where $L=\|A-B\|_{2}$ is the length of the $A B$ segment. When an orthogonal projection is outside the $A B$ segment, $\beta=0$.

\section{REFERENCES}

[1] J. R. Lakowicz, Principles of Fluorescence Spectroscopy. Springer, 2006.

[2] K. Suhling et al., "Fluorescence lifetime imaging (FLIM): Basic concepts and some recent developments," Medical Photonics, vol. 27, pp. 3-40, 2015.

[3] Okabe, K., Inada, N., Gota, C. et al. "Intracellular temperature mapping with a fluorescent polymeric thermometer and fluorescence lifetime imaging microscopy," Nat. Commun., vol. 3, no. 705, 2012

[4] K. Awasthi, D. Moriya, T. Nakabayashi, L. Li, and N. Ohta, "Sensitive detection of intracellular environment of normal and cancer cells by autofluorescence lifetime imaging," J. Photochem. Photobiol. B, vol. 165, pp. 256-265, Dec 2016.

[5] S. P. Poland, N. Krstajić, J. Monypenny et al., "A high speed multifocal multiphoton fluorescence lifetime imaging microscope for live-cell FRET imaging," Biomed. Opt. Express, vol. 6, pp. 277-296, 2015

[6] Y. Long, Y. Stahl, S. Weidtkamp-Peters, et al. "In vivo FRET-FLIM reveals cell-type-specific protein interactions in Arabidopsis roots," Nature, vol.548, pp. 97-102, Jul. 2017.

[7] W. Becker, "Advanced Time-Correlated Single Photon Counting Techniques. " Springer, 2005.

[8] S. Ranjit, L. Malacrida, D. M. Jameson, and E. Gratton, "Fit-free analysis of fluorescence lifetime imaging data using the phasor approach," Nat. Protoc., vol. 13, no. 9, pp. 1979-2004, Sep. 2018.

[9] C. Stringari, A. Cinquin, O. Cinquin, M. A. Digman, P. J. Donovan, and E. Gratton, "Phasor approach to fluorescence lifetime microscopy distinguishes different metabolic states of germ cells in a live tissue," Proc. Natl. Acad. Sci. USA, vol. 108, no. 33, pp. 13582-7, Aug 162011.

[10] D. D. Li et al., "Video-rate fluorescence lifetime imaging camera with CMOS single-photon avalanche diode arrays and high-speed imaging algorithm," J. Biomed. Opt., vol. 16, no. 9, p. 096012, Sep. 2011.

[11] C. Zhang, P. Li, G. Sun, Y. Guan, B. Xiao, and J. Cong, "Optimizing FPGA-based Accelerator Design for Deep Convolutional Neural Networks," presented at the Proceedings of the 2015 ACM/SIGDA Int. Symp. Field-Programmable Gate Arrays - FPGA '15, 2015.

[12] Y. LeCun, Y. Bengio, and G. Hinton, "Deep learning," Nature, vol. 521, no. 7553, pp. 436-44, May 2015.

[13] Ian Goodfellow, Yoshua Bengio, and Aaron Courville, Deep Learning. MIT Press, 2016.

[14] A. Krizhevsky, S. Ilya, and H. G. E, "ImageNet Classification with Deep Convolutional Neural Networks," presented at the NIPS, 2012.

[15] C. Szegedy et al., "Going Deeper with Convolutions," in 2015 Ieee Conference on Computer Vision and Pattern Recognition (IEEE Conference on Computer Vision and Pattern Recognition, New York: Ieee, 2015, pp. 1-9.

[16] K. M. He, X. Y. Zhang, S. Q. Ren, J. Sun, and Ieee, "Deep Residual Learning for Image Recognition," in 2016 Ieee Conference on Computer Vision and Pattern Recognition, New York, 2016, pp. 770-778. 
[17] J. Ma, R. P. Sheridan, A. Liaw, G. E. Dahl, and V. Svetnik, "Deep neural nets as a method for quantitative structure-activity relationships," J. Chem. Inf. Model., vol. 55, no. 2, pp. 263-74, Feb. 2015.

[18] M. Helmstaedter, K. L. Briggman, S. C. Turaga, V. Jain, H. S. Seung, and W. Denk, "Connectomic reconstruction of the inner plexiform layer in the mouse retina," Nature, vol. 500, no. 7461, pp. 168-74, Aug. 2013.

[19] H. Wang et al., "Deep learning enables cross-modality super-resolution in fluorescence microscopy," Nat. Methods., vol. 16, no. 1, pp. 103-110, Jan. 2019.

[20] C. Belthangady and L. A. Royer, "Applications, promises, and pitfalls of deep learning for fluorescence image reconstruction," Nat. Methods., vol. 16, no. 12, pp. 1215-1225, Dec. 2019.

[21] G. Wu, T. Nowotny, Y. Zhang, H. Q. Yu, and D. D. Li, "Artificial neural network approaches for fluorescence lifetime imaging techniques," Opt Lett, vol. 41, no. 11, pp. 2561-4, Jun. 2016.

[22] J. T. Smith et al., "Fast fit-free analysis of fluorescence lifetime imaging via deep learning," Proc. Natl. Acad. Sci. USA, vol. 116, no. 48, pp. 24019-24030, Nov. 2019.

[23] R. Yao, M. Ochoa, P. Yan, and X. Intes, "Net-FLICS: fast quantitative wide-field fluorescence lifetime imaging with compressed sensing - a deep learning approach," Light. Sci. Appl., vol. 8, p. 26, 2019.

[24] U. R. Acharya et al., "A deep convolutional neural network model to classify heartbeats," Comput. Biol. Med., vol. 89, pp. 389-396, Oct. 2017.

[25] S. Kiranyaz, O. Avci, O. Abdeljaber, T. Ince, M. Gabbouj, and D. J. Inman, "1D Convolutional Neural Networks and Applications - A Survey," arXiv:1905.03554 2019.

[26] M. Bettoni, G. Urgese, Y. Kobayashi, E. Macii, and A. Acquaviva, "A Convolutional Neural Network Fully Implemented on FPGA for Embedded Platforms," presented at the 2017 New Generation of CAS (NGCAS), 2017.

[27] S. Ioffe and C. Szegedy, "Batch Normalization: Accelerating Deep Network Training by Reducing Internal Covariate Shift," arXiv: 1502.031672015.

[28] https://www.tensorflow.org/

[29] H. C. Gerritsen, M. A. Asselbergs, A. V. Agronskaia, and W.G. Van Sark. "Fluorescence lifetime imaging in scanning microscopes: acquisition speed, photon economy and lifetime resolution." J. Microsc. vol. 206, pp. 218-24, June. 2002.

[30] Y. Chen, Y. Zhang, D. J. S. Birch and A. S. Barnard, "Creation and luminescence of size selected gold nanorods," Nanoscale, Vol. 4, pp. 5017-5022, Jun. 2012

[31] Y. Zhang, D. J. S. Birch, and Y. Chen, "Two-photon excited surface plasmon enhanced energy transfer between between DAPI and gold nanoparticles: Opportunities in intra-cellar imaging and sensing," Appl. Phys. Lett. vol. 99, 103701, Jun. 2011

[32] G. Wei, J. Yu, J. Wang, P. Gu, D. J. Birch, and Y. Chen, "Hairpin DNA-functionalized gold nanorods for mRNA detection in homogenous solution," J. Biomed. Opt., vol. 21, no. 9, p. 97001, Sep. 2016.

[33] Y. Zhang, G. Wei, J. Yu, D. J. Birch, and Y. Chen, "Surface plasmon enhanced energy transfer between gold nanorods and fluorophores: application to endocytosis study and RNA detection," Faraday Discuss, vol. 178, pp. 383-94, 2015.

[34] Y. Zhang, J. Yu, D. J. S. Birch and Y. Chen, "Gold nanorods for fluorescence lifetime imaging in biology," J. Biomed. Opt., Vol.15, no.2, 020504, Mar. 2010

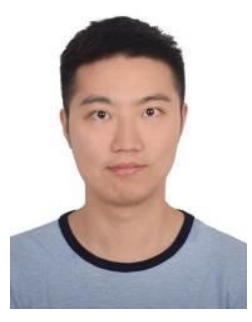

Dong Xiao received the M.S. degree in optics from the Shenzhen University, Shenzhen, China, in 2016. He is currently working toward the Ph.D. degree funded by Medical Research Scotland and Photon Force, Ltd., Edinburgh with the Strathclyde Institute of Pharmacy and Biomedical Sciences, University of Strathclyde, Glasgow, U.K. His current research interests include the fluorescence lifetime imaging microscopy systems, flow cytometry, biomedical image processing, and plasmonics.

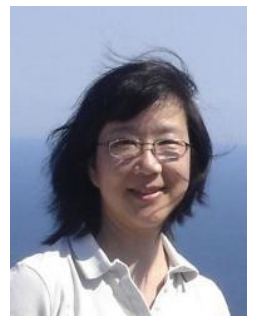

Yu Chen received the Ph.D. degree in nanoscale physics from Birmingham University, Birmingham, U.K., in 2000. Her then was appointed to a lectureship in molecular nanometrology at University of Strathclyde, Glasgow, U.K., in 2007 funded by an EPSRC Science and Innovation Award. She was a senior lecturer from 2013 to 2017 and became a Reader in 2018.

Her main research activities lie in the creation and characterization of nanoscale structures for their unique physical and chemical properties utilizing optical and electron microscopy and spectroscopy. Her recent research focuses on fluorescent nanoclusters, surface plasmon enhanced effects, including two-photon luminescence, energy transfer and SERRS from noble metal nanoparticles, arrays and porous media, with strong links to biomedical imaging and sensing, as well as nanoparticle-cell interaction and cytotoxicity. Other work includes 3D visualization of nanoparticles, stability of Au nanoparticles, and creating nanostructures using chemical lithography and direct writing.

She is a fellow of the Royal Microscopical Society, a member of the European Microscopy Society, the Institute of Physics, and the American Chemical Society. She serves as an expert panel member for the European Cooperation in Science and Technology, a referee for top international journals, an invited speaker and a committee member for a number of international conferences and workshops.

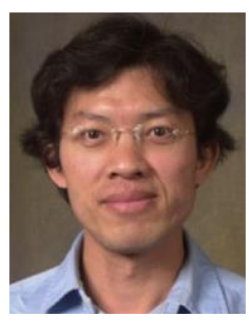

David Day-Uei Li received the Ph.D. degree in electrical engineering from the National Taiwan University, Taipei, Taiwan, in 2001. He then joined the Industrial Technology Research Institute, Taiwan, working on CMOS optical and wireless communication chipsets. From 2007 to 2011, he worked at the University of Edinburgh, Edinburgh, on two European projects focusing on CMOS single-photon avalanche diode sensors and systems. He then took the lectureship in biomedical engineering at the University of Sussex, Brighton, in mid-2011, and in 2014 he joined the University of Strathclyde, Glasgow, as a Senior Lecturer. He has authored more than 80 journal and conference papers and holds 12 patents. His research interests include CMOS sensors and systems, mixed signal circuits, embedded systems, optical communications, FLIM systems and analysis, and field-programmable gate array/graphics processing unit computing. His research exploits advanced sensor technologies to reveal low-light but fast biological phenomena. 